Relations industrielles

Industrial Relations

\title{
The Canadian Labour Market, Readings in Manpower Economics, by Arthur Kruger and Noah M. Meltz, Toronto, Centre for Industrial Relations, University of Toronto, 1968, 312 pp.
}

\section{Bertrand Belzile}

Volume 27, numéro 4, 1972

URI : https://id.erudit.org/iderudit/028342ar

DOI : https://doi.org/10.7202/028342ar

Aller au sommaire du numéro

Éditeur(s)

Département des relations industrielles de l'Université Laval

ISSN

0034-379X (imprimé)

1703-8138 (numérique)

Découvrir la revue

Citer ce compte rendu

Belzile, B. (1972). Compte rendu de [The Canadian Labour Market, Readings in Manpower Economics, by Arthur Kruger and Noah M. Meltz, Toronto, Centre for Industrial Relations, University of Toronto, 1968, 312 pp.] Relations industrielles / Industrial Relations, 27(4), 786-788.

https://doi.org/10.7202/028342ar

Tous droits réservés @ C Département des relations industrielles de l'Universite Laval, 1972
Ce document est protégé par la loi sur le droit d'auteur. L'utilisation des services d'Érudit (y compris la reproduction) est assujettie à sa politique d'utilisation que vous pouvez consulter en ligne.

https://apropos.erudit.org/fr/usagers/politique-dutilisation/ 
se divise en deux parties: les cinq premiers chapitres sont d'ordre analytique et appliquent les outils conventionnels de l'analyse de marché à l'étude des différentes facettes du facteur de production, travail. Les deux derniers chapitres initient le lecteur aux problèmes des relations industrielles proprement dites.

En traitant de multiples aspects de façon concise, l'auteur soutient du début à la fin l'intérêt du lecteur. Ce livre a cependant le défaut de ses qualités. Comme la majorité des manuels introductifs, ce livre tend à donner la fausse impression de solutionner les problèmes au lieu de laisser le lecteur sur des interrogations qui l'inciteraient à poursuivre une réflexion. Il y a cependant une exception, lors de la discussion des courbes Phillips et des attentes des agents économiques sur le niveau des prix futurs. Ici l'auteur insiste sur l'existence d'une controverse sur le sujet et la nécessité de meilleurs travaux théoriques et empiriques. Ecrit avant août 1972, ce livre n'aborde pas une question d'actualité, soit les implications des différentes politiques de revenu.

En résumé, un excellent manuel introductif à l'économique du travail.

\section{Gérard BELANGER}

\section{The Canadian Labour Market, Readings} in Manpower Economics, by Arthur Kruger and Noah M. Meltz, Toronto, Centre for Industrial Relations, University of Toronto, 1968, 312 pp.

The Canadian Labour Market est un livre de lectures choisies. Mais, à l'encontre de la plupart des livres de ce genre, les textes n'ont pas été extraits de livres ou de revues; ils ont été préparés expressément pour être publiés dans ce livre. Les études ont donc le grand avantage d'être originales et de très bien s'intégrer les unes aux autres pour former un ensemble cohérent. De plus, le choix des sujets a été fait de façon très judicieuse, en ce sens que chaque sujet revêt une importance très grande dans le marché du travail canadien. Chaque sujet sera d'ailleurs recensé brièvement dans le texte qui suit, ceci dans l'ordre où chacun apparaît dans le livre.

Micro-economic Theory, Labour Allocation and Manpower Policy, par Arthur Kruger.
La fonction primordiale du marché du travail, comme de tout marché économique d'ailleurs, consiste à allouer les travailleurs aux différents emplois dans l'économie. Dans l'économie de marché canadienne, cette allocation se fait principalement par le mécanisme des prix, le prix du travail s'appelant salaire. Kruger a donc raison d'expliquer, dans ce texte, comment l'économique, et en particulier la micro-économique, facilite la compréhension de cette allocation du travail. L'auteur présente de façon simple et en même temps claire le rôle de l'offre et de la demande du travail, dans la détermination des salaires et des niveaux d'emploi dans les divers marchés du travail. Il souligne cependant deux imperfections, parmi d'autres, qui empêchent les marchés du travail de fonctionner tel que le prédit la théorie économique. $\mathrm{La}$ première imperfection provient du pouvoir des parties en présence; l'autre de la mobilité imparfaite des travailleurs. Tout au long de son texte, l'auteur suggère des politiques de main-d'œuvre susceptibles d'améliorer le fonctionnement des marchés du travail. Finalement, le lecteur moins familier avec la micro-économique peut lire avec profit quelques explications sur les principaux concepts de base dans cette partie de l'économique.

The Analysis of the Labour Market in Classical Economics, par Samuel Hollander.

On sait que la plupart des économistes, dits classiques, qui ont étudié le marché du travail l'ont fait en grande partie au niveau de toute l'économie, à partir de Smith (1776) à Cairnes (1874). Ils se sont donc intéressés à la détermination du taux moyen de salaire et au niveau de l'emploi total. Il faut de plus être conscient du fait que la principale théorie classique des salaires se rapportait surtout à l'offre du travail; il s'agit de la théorie de la subsistance. L'auteur en explique l'essentiel. Il fait de même d'une autre théorie classique qui, elle, se situe du côté de la demande, i.e. la théorie du fonds de salaire. Dans la suite, l'auteur essaie d'évaluer la validité de ces deux théories classiques. Puis, il explique les vues des classiques sur les trois questions suivantes : le chômage; la structure des salaires et le rôle du gouvernement dans l'éducation; et finalement leur atti- 
tude vis-à-vis les syndicats. On y retrouve des commentaires fort intéressants.

\section{Technological Progress and Labour Dis- placement, par Yehuda Kotowitz.}

Kotowitz examine, dans ce texte, un sujet fort controversé. D'après la théorie économique, on est porté à croire que le progrès technologique, et en particulier l'automation, entraîne du chômage. Par ailleurs, les faits passés ne semblent pas généralement confirmer les prédictions de cette théorie. D'abord, l'auteur examine la nature de la demande des facteurs de production dans n'importe quelle technologie. Il analyse ensuite comment la technologie et d'autres variables économiques, affectent cette demande. Il distingue aussi les effets sur l'entreprise ou l'industrie d'une part et sur toute l'économie d'autre part. Enfin, après avoir présenté quelques résultats statistiques préliminaires se rapportant aux industries manufacturières, il tente de dégager certaines conclusions relativement aux effets du progrès technologique sur la maind'œuvre.

C'est avec raison que l'auteur situe son analyse à divers degrés d'aggrégation. En effet, on peut constater un déplacement de travailleurs qui peut être accompagné d'une augmentation de leur nombre total: certains travailleurs sont affectés défavorablement, d'autres favorablement. Et l'auteur conclut en écrivant que c'est précisément l'accumulation du capital qui rend possible une croissance simultanée de l'emploi et des salaires. Il faut de plus être conscient du fait que le progrès technologique peut affecter tout autant la distribution du revenu que l'emploi.

Structural Unemployment, par John W. L. Winder.

L'auteur retient la définition à savoir que le chômage structurel provient du manque de l'économie à s'ajuster suffisamment vite à des circonstances changeantes. A cet égard, il analyse l'influence du système de prix et de la demande globale. $\mathrm{Au}$ fond, la classification des types de chômage peut s'effectuer par les causes ou par les remèdes. L'auteur préfère la deuxième. Il est donc tout naturellement amené à discuter assez longuement la fameuse question de l'option (ou relation d'arbitrage) entre le chômage et l'inflation, ceci à l'aide de la non moins fameuse courbe de Phillips. Il est intéressant de noter qu'un déplacement le long de la courbe s'explique généralement par l'inflation causée par la demande, alors qu'un déplacement de la courbe semble pouvoir s'expliquer par l'inflation causée par les coûts. Ce deuxième type d'inflation à son tour s'explique par des imperfections dans le marché du travail. Les politiques de main-d'œuvre visent à corriger ces imperfections, en partie du moins. Et, selon l'auteur, le dénominateur commun, en un certain sens, de ces politiques est l'amélioration de la mobilité des travailleurs. Il étudie donc la mobilité en regard du chômage structurel. Après avoir passé en revue certaines études empiriques sur le sujet, Winder termine son texte en dégageant des conclusions politiques.

\section{Labour Market Information and Analysis in Canada, par Noah M. Meltz.}

Dans un court texte, Meltz présente une classification fort utile des informations disponibles sur la main-d'œuvre au Canada. Il décrit et critique les principales informations et déplore l'insuffisance de certaines, telles que celles portant sur les gains et les occupations, de même que sur la prévision de maind'œuvre.

\section{Labour Markets and Educational Plann- ing, par Ian Drummond et Measuring the Output of Educational Institutions, par David Stager.}

Le premier texte fournit un relevé de quelques techniques de planification en matière d'éducation. L'auteur s'efforce d'établir le lien étroit entre ce type de planification et le marché du travail. Il conduit son analyse en termes de coûts et bénéfices.

Dans le deuxième texte, Stager indique comment on peut mesurer la production des institutions d'enseignement. Il utilise les trois approches suivantes: 1) le développement intellectuel mesuré par les tests d'aptitudes; les services d'enseignement rendus; et finalement les différentiels de salaire. Cette préoccupation semble primordiale, étant donné l'attention grandissante que l'on accorde aux investissements, entre autres sous forme d'éducation, dans le capital humain. 


\section{Appréciation générale}

Ce livre présente d'excellentes lectures qui permettent de mieux comprendre le fonctionnement du marché du travail canadien. Les aspects plutôt théoriques et généraux sont couverts dans les deux premiers textes, alors que, dans les autres, quelques aspects «pratiques 》 et surtout fort intéressants sont analysés par des spécialistes qui ne perdent jamais de vue que le but du livre consiste à mieux faire connaître le fonctionnement du marché du travail.

\section{Bertrand BELZILE}

Le prix de la santé, par Jean-Luc Migué et Gérard Bélanger. Collection Sciences de l'homme et humanisme. Montréal, Editions Hurtubise HMH, 1972, 238 pp.

A la suite de nombreux économistes, dont Klarman aux Etats-Unis et BrunetJailly en France, les coauteurs veulent montrer ce que l'analyse économique peut apporter de contribution à la compréhension de l'industrie des services de santé. Comme l'ouvrage en est un d'économie appliquée, l'exposé de la théorie économique est délibérément limitée aux seuls outils analytiques directement nécessaires à la compréhension des questions soulevées.

Le lecteur pourra reconnaître deux sections distinctes dans ce volume. Les cinq premiers chapitres traitent du comportement des acteurs principaux du monde de la santé : le patient, le médecin et l'hôpital. Dans les quatre autres chapitres, les auteurs s'attachent dans l'ordre au financement du régime au Québec, à la rentabilité des investissements en santé, aux coûts des médicaments et à l'examen du concept de profession.

Les premières pages sont consacrées aux rudiments de la micro-économique, avec un fort accent sur les règles de comportement tirées du «Welfare economics». Ce chapitre introductif vise surtout à présenter le postulat qui fonde la démarche des auteurs, soit le peu de souveraineté du consommateur dans le secteur santé. Ce dernier ne serait pas en mesure d'exprimer ses préférences et serait impuissant «à apprécier l'utilité qu'il retire d'un produit ou d'un service (p. 20). Le reste du chapitre dégage certaines conséquences de ce postulat sur les comportements des médecins.
Les chapitres II et III traitent du manque d'efficacité dans le fonctionnement des hôpitaux et en proposent une explication. Le diagnostic d'inefficacité se fonde sur les recherches empiriques qui ont découvert un niveau d'utilisation inférieur à la capacité (définie par le coût moyen minimum) et sur «la littérature [qui] fourmille de critiques très sévères à l'endroit de la qualité du management des hôpitaux» (p. 45); d'ailleurs l'absence de concurrence entre les hôpitaux et leur caractère non-lucratif rendraient inévitable cette inefficacité. Pour expliquer plus précisément ce phénomène, les auteurs font appel aux hypothèses de Baumol et surtout de Williamson sur les objectifs que recherchent les administrateurs : rémunération et préférence accordée aux budgets de personnel administratif et d'investissement discrétionnaire. Les auteurs croient retrouver ces objectifs chez les administrateurs hospitaliers : recherche du prestige et du confort par le truchement de l'investissement discrétionnaire et mauvais usage du personnel sous ses ordres qui ouvre à l'administrateur la possibilité d'un emploi prestigieux et rémunérateur.

Dans le chapitre IV l'on s'interroge sur l'efficacité du mode actuel de production des services de santé, caractérisée d'une part par la multiplicité des producteurs (médecins) et d'autre part par la concentration élevée des personnels et équipements spécialisés (hôpitaux). A ce propos, les auteurs résument les recommandations de la Commission d'enquête sur la santé et le bien-être social qui visent à l'intégration des producteurs et des institutions; ils analysent ces recommandations à la lumière du concept d'économies d'échelle et des études empiriques sur le sujet.

L'évolution des prix des services de santé est examinée au chapitre $\mathrm{V}$. Cette analyse comporte deux volets. Dans un premier temps, les auteurs critiquent les indices de prix fournis par les publications officielles, puisqu'ils ne tiennent pas compte des changements dans la qualité des services et des variations dans les prix relatifs des inputs et des outputs; cette façon de procéder tend à surestimer la hausse des coûts et à sous-évaluer les gains de productivité. Puis, pour expliquer l'évolution des prix et du volume des services, ils analysent les déterminants de la demande et de l'offre ainsi que 